\title{
Frontal Sinus
}

National Cancer Institute

\section{Source}

National Cancer Institute. Frontal Sinus. NCI Thesaurus. Code C12277.

The paired, mucosal lined air spaces located above the orbit and communicating with the nasal passages. 